\title{
Local Buckling of Steel Plates in Composite Structures under Combined Bending and Compression
}

\author{
Ying $\mathrm{QIN},{ }^{1,2) *}$ Er-Feng DU, ${ }^{1)}$ Yong-Wei $\mathrm{LI}^{1)}$ and Jing-Chen ZHANG ${ }^{1)}$ \\ 1) Key Laboratory of Concrete and Prestressed Concrete Structures of the Ministry of Education, and National Prestress Engi- \\ neering Research Center, Southeast University, Nanjing, 211189 China. \\ 2) State Key Laboratory of Subtropical Building Science, South China University of Technology, Guangzhou, 510641 China.
}

(Received on March 22, 2018; accepted on July 11, 2018; J-STAGE Advance published date: August 30, 2018)

\begin{abstract}
In this paper, explicit local buckling analysis of steel plates in composite structures under combine bending and compression and with elastic restraint against rotation is presented. Unique displacement function, which combines sinusoidal and cosine functions along $x$ - and $y$-directions and satisfies boundary and loading conditions, is proposed to account for the effect of restraint of concrete and the elastic rotational restraint stiffness along both the loaded and unloaded edges of the steel plates. The explicit local buckling solution for the elastically restrained steel plates in composite structures is simplified to several special cases (e.g., the CS, CC, SS and KS steel plates) subjected to combined bending and compression. To verify the accuracy of the explicit solutions, experimental data, available solutions in the literature, and finite element results are compared, and reasonable agreement has been observed, particularly for the simplified cases. Parametric study is further conducted, and the effects of different parameters such as the aspect ratio and the loading stress gradient parameter on the local buckling stress resultants of steel plates with different boundary conditions are discussed.
\end{abstract}

KEY WORDS: local buckling; composite structures; combined compression and bending; steel plate.

\section{Introduction}

Concrete-steel composite structures are effective structural system and are increasingly applied in civil engineering. Concrete-filled tube (CFT) columns, as a typical type of composite structures, are composed of the steel tube constructed by four steel plates and the infilled concrete. The steel plates in CFT columns are subjected to combined bending and compression. Local buckling of steel plates in combined stress states may occur, depending on the boundary condition and plate thickness.

Timoshenko and Gere ${ }^{1)}$ wrote a famous book named Theory of Elastic Stability. Analytical approach to obtain the local buckling of steel plate was introduced. However, the considered boundary condition in this book is only simply-supported or clamped. Furthermore, the steel plate in their research is not in contact with other rigid medium. The existence of concrete will significantly affect the buckling shape of the steel plate.

The research on the local buckling of steel plates has also been conducted by many other researchers. Qiao et al. ${ }^{2)}$ presented an analytical approach to evaluate the local buckling of discrete laminated plates under generic in-plane loading and with two opposite edges simply-supported and the other two opposite edges either both rotationally restrained

\footnotetext{
* Corresponding author: E-mail: qinying@seu.edu.cn

DOI: https://doi.org/10.2355/isijinternational.ISIJINT-2018-202
}

or one rotationally restrained and the other free. Liu et al. ${ }^{3)}$ extended the work by Qiao et $a .^{2)}$ to the case that the steel plates is under combined uniform in-plane shear and linearly varying axial loads. Recently, Dong et al. ${ }^{4)}$ proposed analytical method to obtain the buckling coefficient of symmetrically laminated composite plate under in-plane shear.

In contrast, the local buckling behavior of steel plates in contact with concrete is quite different. The plates are restrained to buckle locally in one lateral direction due to the existence of concrete. The importance of this feature has been investigated by several researchers mostly based on numerical approaches. Uy and Bradford ${ }^{5)}$ applied finite strip method to study the elastic local buckling performance of steel plates in composite steel-concrete members. Liang et $a l .{ }^{6)}$ used finite element method to obtain the local buckling strength of steel plates in concrete-filled steel tubular columns and double skin composite plates.

Although the finite element methods can be used to predict the local buckling strength of steel plates in composite structures with various boundary and loading conditions, ${ }^{7,8)}$ it is time consuming and tedious for general use by civil engineers. Therefore, efforts have been made to obtain the closed-form explicit solutions for steel plates in composite structures with some simply loading and boundary conditions. Wright ${ }^{9)}$ presented an analytical model to predict the local buckling behavior of sections where steel plates were in contact with a rigid medium. Long et al. ${ }^{10)}$ proposed explicit solutions for the local buckling of steel plates in 
CFT columns subjected to eccentric compression and with clamped loaded edges. Qin et al. ${ }^{11)}$ investigated the critical buckling problem of steel plates in CFT columns subjected to uniform compression.

Furthermore, it is important to choose a proper displacement function for the buckling shape when applying Rayleigh-Rize method. Several displacement functions have been given by many researchers for plate with various loading and boundary conditions. Arabzade et al. ${ }^{12)}$ proposed polynomial functions that include kinematic and geometric boundary conditions to construct the displacement function of steel plate under shear force, as given by Eq. (1). Seif and Kabir $^{13)}$ used piecewise functions with minimum degrees of freedom to describe the buckling mode shapes of plate under tension, as given in Eq. (2), where $w_{o}$ is the maximum deflection. These functions were obtained through multiplying an exponential decay that exerts locally the nature of buckling, to polynomial functions. Jaberzadeh and Azhari ${ }^{14)}$ applied combined trigonometric and polynomial functions to satisfy the boundary conditions for stiffened plates under non-uniform compression, as illustrated in Eq. (3). Mittelstedt ${ }^{15)}$ derived a closed-form solution for a uniaxially loaded clamped stringer plate. A double series expansion using simple sin-functions in both inplane directions was employed as the buckling shape, as shown in Eq. (4). In addition, Mittelstedt ${ }^{16)}$ proposed another type of displacement function given in Eq. (5) to solve the similar problem. Long et al. ${ }^{10)}$ assumed the displacement function for steel plate in the $x$ direction is a cosine function and the deflection function in the $y$ direction is a biquadratic function, as expressed by Eq. (6). Liu et al. $^{3)}$ treated the plate under uniform in-plane shear and linearly varying axial loads as a repeating element and proposed the buckled shape function as given in Eq. (7). Kang and Leissa ${ }^{17)}$ studied the buckling of rectangular plates having linearly varying in-plane loading on two opposite simply-supported edges. By assuming the displacement function in $x$-direction to vary as $\sin (m \pi x / a)$, the function in $y$-direction was simplified to a power series as give in Eq. (8). Qiao et al. ${ }^{2)}$ used the combined polynomial and trigonometric displacement function, as shown in Eq. (9), to predict the plate with two opposite edges simply-supported, one other edge rotationally restrained and the other one free.

$$
\begin{aligned}
& w(\xi, \eta)=\phi_{b}(\xi, \eta) \sum_{i=1}^{n} \sum_{j=1}^{n} \alpha_{i j} f_{i}(\xi) f_{j}(\eta) \\
& w(x, y)= \begin{cases}w_{o}\left[f_{1}(x) g_{1}(y)+f_{2}(x) g_{2}(y)\right] & \text { if } 0 \leq x \leq 1 \\
w_{o}\left[f_{3}(x) g_{2}(y)\right] & \text { if } 1 \leq x \leq \omega\end{cases} \\
& w=\sum_{m} \sum_{n} q_{m n} \sin (m \pi \xi) \\
& \left(\eta^{n+6}+A_{n} \eta^{n+5}+B_{n} \eta^{n+4}+C_{n} \eta^{n+3}+D_{n} \eta^{n+2}+E_{n} \eta^{n+1}+F_{n} \eta^{n}\right) \\
& w=\sum_{m=1}^{m=M} \sum_{n=1}^{n=N} W_{m n} \sin \left(\frac{m \pi x_{1}}{a}\right) \sin \left(\frac{n \pi x_{2}^{p}}{b}\right)
\end{aligned}
$$

$$
\begin{gathered}
w\left(x_{1}, x_{2}\right)=\sum_{m=1}^{m=M} \sum_{n=2}^{n=N+1} W_{m n} \sin \left(\frac{m \pi x_{1}}{a}\right) \times \\
\left\{\cos \left[\frac{(n-2) \pi x_{2}}{b}\right]-\cos \left(\frac{n \pi x_{2}}{b}\right)\right\} \\
w=c\left[a_{1} \frac{y}{b}\left(1-\frac{y}{b}\right)+a_{2}\left(\frac{y}{b}\right)^{2}\left(1-\frac{y}{b}\right)+a_{3}\left(\frac{y}{b}\right)\left(1-\frac{y}{b}\right)^{2}\right. \\
\left.+a_{4}\left(\frac{y}{b}\right)^{2}\left(1-\frac{y}{b}\right)^{2}\right] \times\left(1-\cos \frac{2 \pi x}{a}\right)
\end{gathered}
$$

$$
\begin{array}{r}
w=\left[A_{1} f_{1}(y)+A_{2} f_{2}(y)+A_{3} f_{3}(y)\right] \times \sin \frac{\pi(x-\varphi y)}{a} \\
w(\xi, \eta)=\sum_{n=0}^{\infty} C_{m, n} \eta^{n} \times \sin (m \pi \xi)
\end{array}
$$

$$
\begin{aligned}
& w(x, y)= \\
& w_{0}\left\{\left(1-\omega_{y}\right) \frac{y}{b}+\omega_{y}\left[8-8 \cos \left(\frac{\pi y}{2 b}\right)+\sin \left(\pi \frac{y}{b}\right)-\pi \frac{y}{b}\right]\right\} \sin \frac{\pi x}{a}
\end{aligned}
$$

This paper investigated the case that the steel plates in contact with concrete are subjected to combined bending and compression and with different boundary conditions. The energy methods are applied and the unique displacement function which considers the effect of concrete is proposed by combining sinusoidal and cosine functions along both $x$ and $y$-directions. Explicit local buckling solutions for several different boundary conditions are discussed and the present results are validated against both the available solutions in the literature and the finite element eigenvalue solutions. A parametric study is further conducted to investigate the influence of several factors such as the loading stress gradient parameter and the aspect ratio on the local buckling stress resultants of the steel plates in composite structures.

\section{Analytical Formulation}

\subsection{Formulation for Elastically-Restrained Steel Plates}

A representative unit of a steel plate in composite structures is considered and depicted in Fig. 1. The steel plate is elastically restrained along four edges, with a length of $a$ and a width of $b$. The steel plate is subjected to combined bending and compression, and is represented by eccentric

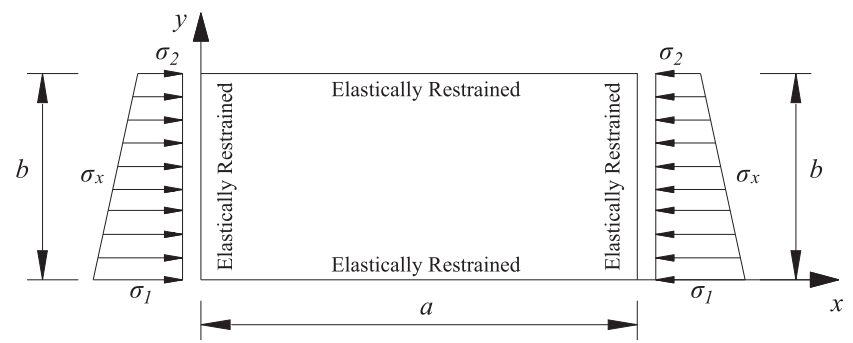

Fig. 1. Geometry of elastically restrained steel plate under combined bending and compression. 
compression $\sigma_{x}$ as expressed in Eq. (10).

$$
\sigma_{x}=\sigma_{1}\left(1-\varsigma \frac{y}{b}\right)
$$

where $\varsigma$ is the loading stress gradient parameter along the $y$-direction and is given by Eq. (11). It should be noted that $\varsigma=0$ means the steel plate is under uniaxial compression along the $x$-direction.

$$
\varsigma=\frac{\sigma_{1}-\sigma_{2}}{\sigma_{1}}
$$

The energy method is used to evaluate the elastic local buckling of the steel plates with the elastic restraint and subjected to combined bending and compression. The total potential energy of the steel plate system $\Pi$ is composed of the elastic strain energy stored in the deformed steel plate $U$, the restraining energy stored in the rotational springs along the loaded and unloaded edges $U_{\Gamma}$, and the work done by the external bending and compression $V$, and they can be expressed, respectively, as

$$
\Pi=U+U_{\Gamma}+V
$$

$$
\begin{aligned}
& U= \\
& \frac{D}{2} \iint_{\Omega}\left\{\left(\frac{\partial^{2} w}{\partial x^{2}}+\frac{\partial^{2} w}{\partial y^{2}}\right)^{2}-2(1-v)\left[\frac{\partial^{2} w}{\partial x^{2}} \frac{\partial^{2} w}{\partial y^{2}}-\left(\frac{\partial^{2} w}{\partial x \partial y}\right)^{2}\right]\right\} d x d y \\
& U_{\Gamma}=\frac{1}{2} \int_{\Gamma x} k_{y}\left(\left.\frac{\partial w}{\partial y}\right|_{y=0}\right)^{2} d x+\frac{1}{2} \int_{\Gamma x} k_{y}\left(\left.\frac{\partial w}{\partial y}\right|_{y=b}\right)^{2} d x \\
& +\frac{1}{2} \int_{\Gamma y} k_{x}\left(\left.\frac{\partial w}{\partial x}\right|_{x=0}\right)^{2} d y+\frac{1}{2} \int_{\Gamma y} k_{x}\left(\left.\frac{\partial w}{\partial x}\right|_{x=a}\right)^{2} d y \\
& V=\frac{1}{2} \iint_{\Omega} N_{x}\left(\frac{\partial w}{\partial x}\right)^{2} d x d y
\end{aligned}
$$

where $D$ is the flexural rigidity of the steel plate and is given by Eq. (16); $\Omega$ is the area of the steel plate; $v$ is the Poisson's ratio; $w$ is the displacement function of the steel plate; $E$ is the elastic modulus of steel; $t$ is the thickness of steel plate; $k_{x}, k_{y}$ are the elastic rotational stiffness of springs at the loaded edges and unloaded edges, respectively; $\Gamma_{x}, \Gamma_{y}$ are the boundary lines along the unloaded edges $(y=0$ and $y=a)$ and loaded edges $(x=0$ and $x=b)$, respectively; and $N_{x}=\sigma_{x} t$ is the axial load per unit length at the boundary line along the loaded edges.

$$
D=\frac{E t^{3}}{12\left(1-v^{2}\right)}
$$

By substituting the appropriate out-of-displacement buckling shape into Eqs. (13)-(15) and the corresponding results into Eq. (12), the buckling eigenvalue problem can be obtained by Rayleigh-Rize method. The detail of Eqs. (12)(15) can be found in the standard textbooks and for brevity, they are not elaborated.

\subsection{Displacement Formulations}

An adequate and efficient set of displacement function has to be formulated for steel plates in composite structures to obtain an effective approach to the explicit local buckling solution. Furthermore, the chosen displacement function should be as simple as possible to reduce the computational complexity to a reasonable level and to derive the closedform solution that can be easily used in daily engineering practice. From the literature review it can be observed that many of the proposed displacement functions by previous research are with too many variables to be determined, which will increase the complexity to solve the explicit solution. Furthermore, previous displacement functions cannot satisfy both loading and boundary conditions for steel plate in contact with concrete and subjected to combined compression and bending.

For the considered steel plate with elastic restraint, the following boundary conditions should be satisfied. Firstly, the displacement function $w$ along the four edges should be zero, as shown in Eqs. (17a) and (17d). Secondly, the bending moment along the edges should meet the requirements given in Eqs. (17b), (17c), (17e), and (17f).

$$
\begin{aligned}
& w(0, y)=w(a, y)=0 \text {. } \\
& M_{x}(0, y)=-D\left(\frac{\partial^{2} w}{\partial x^{2}}\right)_{x=0}=-k_{x}\left(\frac{\partial w}{\partial x}\right)_{x=0} \\
& M_{x}(a, y)=-D\left(\frac{\partial^{2} w}{\partial x^{2}}\right)_{x=a}=+k_{x}\left(\frac{\partial w}{\partial x}\right)_{x=a} \\
& w(x, 0)=w(x, b)=0 \\
& M_{y}(x, 0)=-D\left(\frac{\partial^{2} w}{\partial y^{2}}\right)_{y=0}=-k_{y}\left(\frac{\partial w}{\partial y}\right)_{y=0} \\
& M_{y}(x, b)=-D\left(\frac{\partial^{2} w}{\partial y^{2}}\right)_{y=b}=+k_{y}\left(\frac{\partial w}{\partial y}\right)_{y=b}
\end{aligned}
$$

Meanwhile, when considering the buckling behavior of such a steel plate in touch with concrete, the only possibility of buckling shape is to buckle away from the concrete. For the sake of simplicity, the buckling shape function should include proper numbers of unknown coefficients in order to derive a closed-form solution. Furthermore, it may be safe to assume that one half-wave is developed along both direction of the steel plate. Therefore, the unique combination of weighted sinusoidal and cosine functions along the $x$ - and $y$-directions, respectively, are chosen as the buckling shape functions, as given by,

$$
\begin{aligned}
w(x, y) & =C\left[\left(1-\omega_{1}\right) \sin \frac{\pi y}{b}+\omega_{1}\left(1-\cos \frac{2 \pi y}{b}\right)\right] \\
& \times\left[\left(1-\omega_{2}\right) \sin \frac{\pi x}{a}+\omega_{2}\left(1-\cos \frac{2 \pi x}{a}\right)\right]
\end{aligned}
$$

where $C$ is a constant, and $\omega_{1}$ and $\omega_{2}$ are the weight constants and can be determined by the requirement for boundary conditions and compatibility. By appropriately choosing the value of $\omega_{1}$ and $\omega_{2}$, the buckling shape offer reasonable results to reflect the elastic restraint along both loaded and unloaded edges. As can be seen from Fig. 2, $\omega_{1}=0$ cor- 


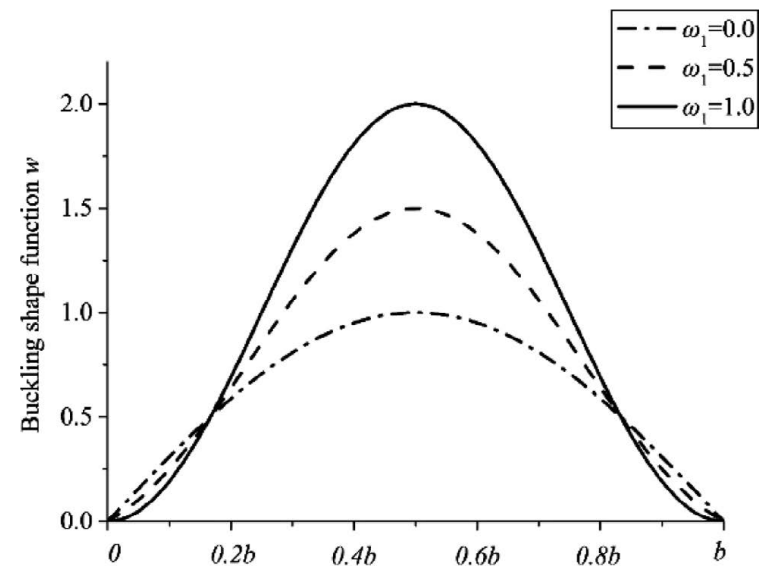

Fig. 2. Buckling shapes with different $\omega$ values.

responds to the case that the edge of steel plate is simplysupported, while $\omega_{1}=1$ corresponds to the case that the edge of steel plate is clamped. Any value of $\omega_{1}$ between 0 and 1 implies that the steel plate is elastically restrained against rotation along the edge.

It should be noted that Eq. (18) exactly satisfy the edge conditions as specified in Eqs. (17a) and (17d). Substituting the first-order partial derivative and the second-order partial derivative of Eq. (18) with respect to $x$ into Eq. (17b) or (17c) gives the weight constant $\omega_{2}$ in terms of the rotational stiffness of the elastic restraint $\left(k_{x}\right)$ along the loaded edges:

$$
\omega_{2}=\frac{k_{x} a}{k_{x} a+4 \pi D}
$$

Substituting the derivative function of Eq. (18) with respective to $y$ into Eqs. (17e) or (17f), the weight constants $\omega_{1}$ can be solved in terms of the elastic rotational restraint stiffness $\left(k_{y}\right)$ along the unloaded edges as

$$
\omega_{1}=\frac{k_{y} b}{k_{y} b+4 \pi D}
$$

Substituting Eq. (18) into Eqs. (13)-(15) and integrating gives,

$$
\begin{aligned}
& U=\frac{D}{2}\left[\begin{array}{l}
\frac{b \pi^{2} C^{2}}{36 a^{3}} A_{1} B_{1}+\frac{a \pi^{2} C^{2}}{36 b^{3}} A_{2} B_{2} \\
+\frac{v+(1-v) \pi}{18 a b} \pi C^{2} A_{3} B_{3}
\end{array}\right] \\
& U_{\Gamma}=\frac{k_{y} a \pi\left(1-\omega_{1}\right)^{2} C^{2}}{6 b^{2}} B_{2}+\frac{k_{x} b \pi\left(1-\omega_{2}\right)^{2} C^{2}}{6 a^{2}} A_{1} \ldots \\
& V=-\frac{\sigma_{1} t b C^{2}}{144 a} A_{4} B_{3}
\end{aligned}
$$

where $A_{1}, A_{2}, A_{3}, A_{4}, B_{1}, B_{2}$ and $B_{3}$ are defined as

$$
\begin{gathered}
A_{1}=(12 \pi-32) \omega_{1}^{2}+(32-6 \pi) \omega_{1}+3 \pi \ldots \\
A_{2}=(51 \pi-32) \omega_{1}^{2}+(32-6 \pi) \omega_{1}+3 \pi \ldots \\
A_{3}=(15 \pi-32) \omega_{1}^{2}+(32-6 \pi) \omega_{1}+3 \pi \ldots \\
A_{4}=\left(32 \omega_{1}^{2}-12 \pi \omega_{1}^{2}+6 \pi \omega_{1}-32 \omega_{1}-3 \pi\right) \varsigma \\
\quad+24 \pi \omega_{1}^{2}-64 \omega_{1}^{2}-12 \pi \omega_{1}+64 \omega_{1}+6 \pi
\end{gathered}
$$

$$
\begin{aligned}
& B_{1}=(51 \pi-32) \omega_{2}^{2}+(32-6 \pi) \omega_{2}+3 \pi \\
& B_{2}=(12 \pi-32) \omega_{2}^{2}+(32-6 \pi) \omega_{2}+3 \pi \\
& B_{3}=(15 \pi-32) \omega_{2}^{2}+(32-6 \pi) \omega_{2}+3 \pi
\end{aligned}
$$

\subsection{Explicit Solution}

Inserting Eqs. (21a)-(21c) into Eq. (12) leads to the following explicit expression for the total potential energy of the steel plate:

$$
\begin{aligned}
\Pi= & \frac{D}{2}\left[\begin{array}{c}
\frac{b \pi^{2} C^{2}}{36 a^{3}} A_{1} B_{1}+\frac{a \pi^{2} C^{2}}{36 b^{3}} A_{2} B_{2} \\
+\frac{v+(1-v) \pi}{18 a b} \pi C^{2} A_{3} B_{3}
\end{array}\right]-\frac{\sigma_{1} t b C^{2}}{144 a} A_{4} B_{3} \\
& +\frac{k_{y} a C^{2}}{2 b^{2}}\left(1+A_{6}^{2}\right) B_{5}+\frac{k_{x} b \pi^{2} C^{2}(1-\omega)^{2}}{a^{2}} A_{7}
\end{aligned}
$$

The fundamental relation that governs the buckling shape of the steel plate states that the first-order derivative of the potential energy $\Pi$ in the buckled state has to vanish:

$$
\frac{\partial \Pi}{\partial C}=0
$$

Substituting Eq. (23) into Eq. (24) leads to the following governing equation for the critical buckling stress:

$$
\begin{aligned}
\sigma_{x} t & =\frac{\pi^{2} D}{b^{2}}\left[\begin{array}{c}
\frac{2 A_{1} B_{1}}{\gamma^{2} A_{4} B_{3}}+\frac{2 \gamma^{2} A_{2} B_{2}}{A_{4} B_{3}}+\frac{4 v A_{3}+4(1-v) \pi A_{3}}{\pi A_{4}} \\
+\frac{48 \lambda_{y} \gamma^{2}\left(1-\omega_{1}\right)^{2} B_{2}}{\pi A_{4} B_{3}}+\frac{48 \lambda_{x}\left(1-\omega_{2}\right)^{2} A_{1}}{\gamma^{2} \pi A_{4} B_{3}}
\end{array}\right] \\
& =\frac{k \pi^{2} D}{b^{2}}
\end{aligned}
$$

where $\gamma$ is the aspect ratio and is defined as $\gamma=a / b ; k$ is elastic local buckling coefficient of the steel plate as given in Eq. (26); and $\lambda_{x}$ and $\lambda_{y}$ are elastically restraining factors of loaded and unloaded edges, respectively, as defined in Eqs. (27a)-(27b).

$$
\begin{aligned}
k= & \frac{2 A_{1} B_{1}}{\gamma^{2} A_{4} B_{3}}+\frac{2 \gamma^{2} A_{2} B_{2}}{A_{4} B_{3}}+\frac{4 v A_{3}+4(1-v) \pi A_{3}}{\pi A_{4}} \\
& +\frac{48 \lambda_{y} \gamma^{2}\left(1-\omega_{1}\right)^{2} B_{2}}{\pi A_{4} B_{3}}+\frac{48 \lambda_{x}\left(1-\omega_{2}\right)^{2} A_{1}}{\gamma^{2} \pi A_{4} B_{3}}
\end{aligned}
$$

$$
\begin{aligned}
& \lambda_{x}=\frac{k_{x} a}{2 D} \\
& \lambda_{y}=\frac{k_{y} b}{2 D}
\end{aligned}
$$

By taking a partial derivative of Eq. (26) with respect to $\gamma$ and setting the derivative equal to zero, the governing critical elastic local buckling coefficient $k_{c r}$ can be obtained. The corresponding critical aspect ratio $\gamma_{c r}$ can be determined by, 


$$
\gamma_{c r}=\left[\frac{\pi A_{1} B_{1}+24 \lambda_{x}\left(1-\omega_{2}\right)^{2} A_{1}}{\pi A_{2} B_{2}+24 \lambda_{y}\left(1-\omega_{1}\right)^{2} B_{2}}\right]^{\frac{1}{4}}
$$

Substituting Eq. (28) into Eq. (26), the critical elastic local buckling coefficient $k_{c r}$ can be determined. Then the critical local buckling stress of steel plate $\sigma_{c r}$ is calculated by substituting $k_{c r}$ and $D$ into Eq. (25) as

$$
\sigma_{c r}=\frac{k_{c r} \pi^{2} E}{12\left(1-v^{2}\right)(b / t)^{2}}
$$

\section{Verification}

In this section, the explicit formulas for local buckling of steel plate in composite structures for several special cases that are commonly used in the practical design are reduced from Eq. (26) and discussed in detail. It is noted that the test results and numerical solutions for some simplified cases are available in the literature, which could indirectly verify the accuracy of the proposed explicit solutions.

Meanwhile, finite element simulations are conducted by the commercial program ANSYS to further validate the present method. The considered material properties of the steel plates are: $E=2.06 \times 10^{5} \mathrm{~N} / \mathrm{mm}^{2}, v=0.3$. In the modelling herein, the steel plate was modelled by the 8 -node shell elements SHELL93, and the concrete was modelled by the 8-node solid structural elements SOLID65. The interface between steel plate and concrete was simulated by creating contact pairs with the 3-D target surface elements TARGE170 and the 3-D 8-node surface-to-surface contact elements CONTA174. The rotational restraints were modelled by a series of spring elements COMBIN14 connected to the nodes along the edges of steel plate.

As noticed in this study, a steel plate with different boundary conditions is discussed, and the notations of CS, $\mathrm{CC}, \mathrm{CK}, \mathrm{KS}$, and $\mathrm{KK}$ steel plates are used to represent the restraining effects along the four edges. The first letter stands for the boundary condition for the loaded edges, while the second letter corresponds to the boundary condition for the unloaded edges. The symbols $\mathrm{C}, \mathrm{S}$, and $\mathrm{K}$ represent the clamped, simply-supported and elastically restrained edges, respectively.

\subsection{CS Steel Plate}

The CS steel plate is clamped along the loaded edges while simply-supported along the unloaded edges. The boundary condition of clamped loaded edges at $x=0$ and $a$ corresponds to $\omega_{2}=1$, while that of simple supported unloaded edges at $y=0$ and $b$ corresponds to $\omega_{1}=0$. The value of critical aspect ratio $\gamma_{c r}=1.52$ can then be obtained by Eq. (28). Substituting $\gamma_{c r}=1.52$ into Eq. (26) gives the value of critical local buckling coefficient $k_{c r}$ as,

$$
k_{c r}=\frac{5.06}{1-0.5 \varsigma}
$$

Particularly, when $\varsigma=0$, the critical local buckling stress of the CS steel plate under uniform compression can be reduced to

$$
\sigma_{c r}=\frac{5.06 \pi^{2} E}{12\left(1-v^{2}\right)(b / t)^{2}}
$$

This results is close to $k_{c r}=5.6$ proposed by $\mathrm{Uy}$ and Bradford ${ }^{5)}$ based on finite strip method.

To verify the prediction accuracy of the present explicit solution about CS steel plate, finite element program ANSYS is conducted to obtain the critical local buckling stress by eigenvalue analysis. A square CS steel plate $(\gamma=$ 1) with the width of $100 \mathrm{~mm}$ and the thickness of $1 \mathrm{~mm}$ is modelled. To simulate the boundary conditions with clamped loaded and simply-supported unloaded edges, while simultaneously to allow translational movement of steel plate along the $x$-direction when subjected to combined compression and bending as shown in Fig. 1, one boundary line along the loaded edge of the steel plate $(x=0)$ is completely restrained against all degrees of freedom, while the other boundary line $(x=a)$ is restrained against all rotational degrees of freedom and $y$ - and $z$-directions translational degrees of freedom. Meanwhile, the two boundary lines along the unloaded edges $(y=0$ and $y=b)$ are restrained against $y$ - and $z$-directions translational degrees of freedom. The typical buckled shape is shown in Fig. 3 .

As indicated in Table 1 and Fig. 4, the present explicit solutions of critical local buckling stress resultants compare closely with numerical predictions. The ratio of the predicted explicit solutions to the numerical ones has a mean value of $93.44 \%$ and a standard deviation of 0.0037 .

\subsection{Steel Plate}

CC steel plate is clamped along both loaded and unloaded

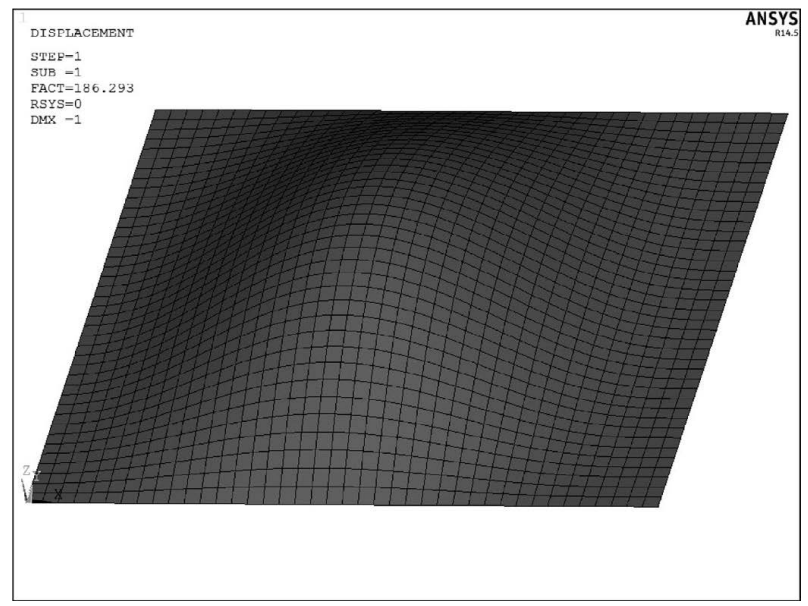

Fig. 3. Typical buckling shape for CS steel plate.

Table 1. Comparison of local buckling stress for CS steel plate.

\begin{tabular}{lccccccc}
\hline \multicolumn{1}{c}{$\varsigma$} & $\begin{array}{c}\text { Explicit } \\
N / \mathrm{mm}^{2}\end{array}$ & $\begin{array}{c}\mathrm{FE} \\
N / \mathrm{mm}^{2}\end{array}$ & $\begin{array}{c}\text { Exp/FE } \\
\%\end{array}$ & $\varsigma$ & $\begin{array}{c}\text { Explicit } \\
N / \mathrm{mm}^{2}\end{array}$ & $\begin{array}{c}\mathrm{FE} \\
N / \mathrm{mm}^{2}\end{array}$ & $\begin{array}{c}\text { Exp/FE } \\
\%\end{array}$ \\
\hline 0 & 117.49 & 126.15 & 93.13 & 0.05 & 120.50 & 129.38 & 93.14 \\
0.15 & 127.01 & 136.34 & 93.16 & 0.25 & 134.27 & 144.15 & 93.15 \\
0.35 & 142.41 & 152.85 & 93.17 & 0.45 & 151.60 & 162.62 & 93.22 \\
0.55 & 162.05 & 173.70 & 93.29 & 0.65 & 174.05 & 186.29 & 93.43 \\
0.75 & 187.98 & 200.90 & 93.57 & 0.85 & 204.33 & 217.92 & 93.76 \\
0.95 & 223.78 & 237.93 & 94.05 & 1.00 & 234.97 & 249.30 & 94.25 \\
\hline
\end{tabular}




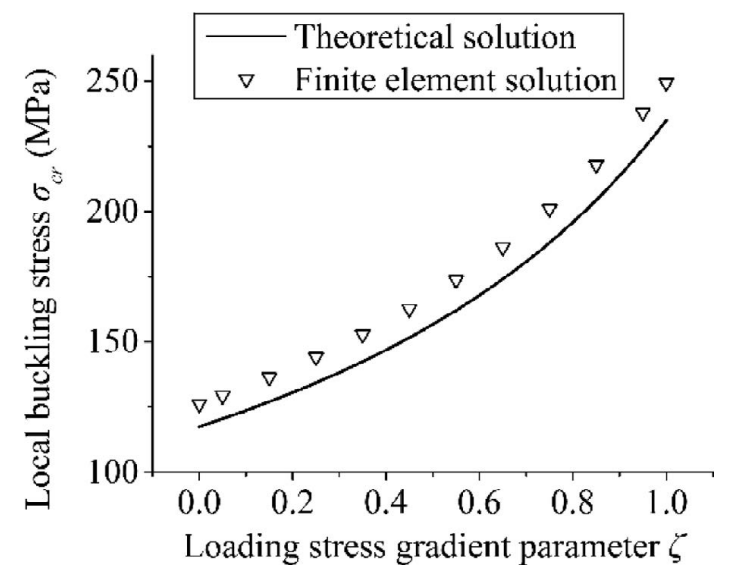

Fig. 4. Comparison of local buckling stress for CS steel plate.

edges. The boundary condition of clamped loaded edges at $x=0$ and $a$ corresponds to $\omega_{2}=1$, while that of clamped unloaded edges at $y=0$ and $b$ corresponds to $\omega_{1}=1$. The value of critical aspect ratio $\gamma_{c r}=1.0$ can then be obtained by Eq. (28). Substituting $\gamma_{c r}=1.0$ into Eq. (26) gives the value of critical local buckling coefficient $k_{c r}$ as,

$$
k_{c r}=\frac{10.12}{1-0.5 \varsigma}
$$

Particularly, when $\varsigma=0$, the critical local buckling stress of the CC steel plate under uniform compression can be simplified as

$$
\sigma_{c r}=\frac{10.12 \pi^{2} E}{12\left(1-v^{2}\right)(b / t)^{2}}
$$

This results is close to $k_{c r}=9.81$ obtained by Liang and $\mathrm{Uy}^{18)}$ and Liang et al. ${ }^{19)}$ by a linear finite element buckling analysis of plates based on the bifurcation buckling theory, $k_{c r}=9.99$ recommended by Bridge and O'Shea ${ }^{20)}$ based on finite strip analysis, $k_{c r}=10.31$ which was proposed by Uy and Bradford, ${ }^{5)}$ and $k_{c r}=10.312$ suggested by Long et al. ${ }^{10)}$

The finite element model was established to simulate the boundary conditions of CC steel plate with clamped both loaded and unloaded edges, one boundary line along the loaded edge of the steel plate $(x=0)$ is completely restrained against all degrees of freedom, while the other loaded boundary line $(x=a)$ and the two unloaded boundary lines $(y=0$ and $y=b)$ are restrained against all rotational degrees of freedom and $y$ - and $z$-directions translational degrees of freedom. The typical buckled shape of a square steel plate ( $b=100 \mathrm{~mm}$ and $t=1 \mathrm{~mm})$ with all edges clamped is illustrated in Fig. 5. The comparison between the proposed explicit and finite element eigenvalue solutions is given in Table 2 and Fig. 6.

It can be observed from Table 2 and Fig. 6 that, in general, the formulas proposed have good accuracy with the numerical solutions. The ratios of the predicted explicit solutions to the numerical ones range from 1.06 to 1.07 with a mean of $106.56 \%$ and a standard deviation of 0.005 . These values closely correspond.

\subsection{SS Steel Plate}

The SS steel plate is simply-supported along both loaded and unloaded edges. This boundary condition corresponds

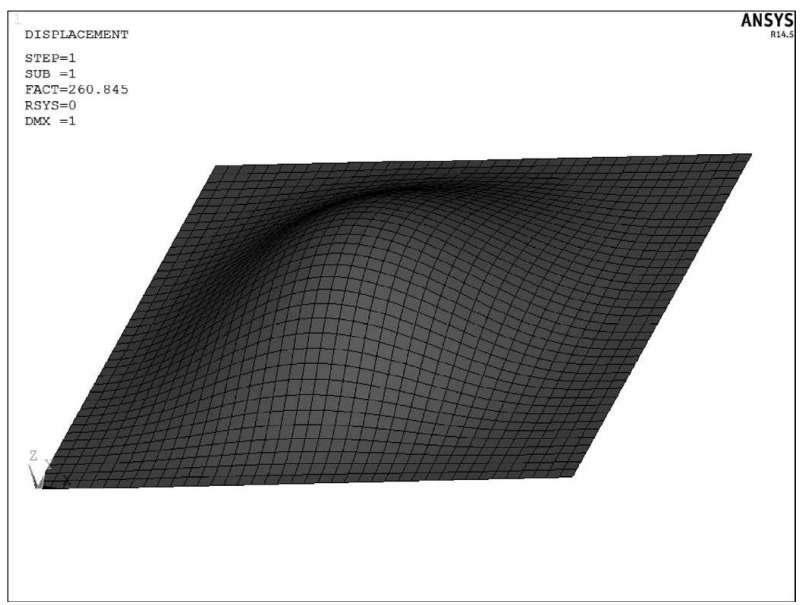

Fig. 5. Typical buckled shape of CC steel plate.

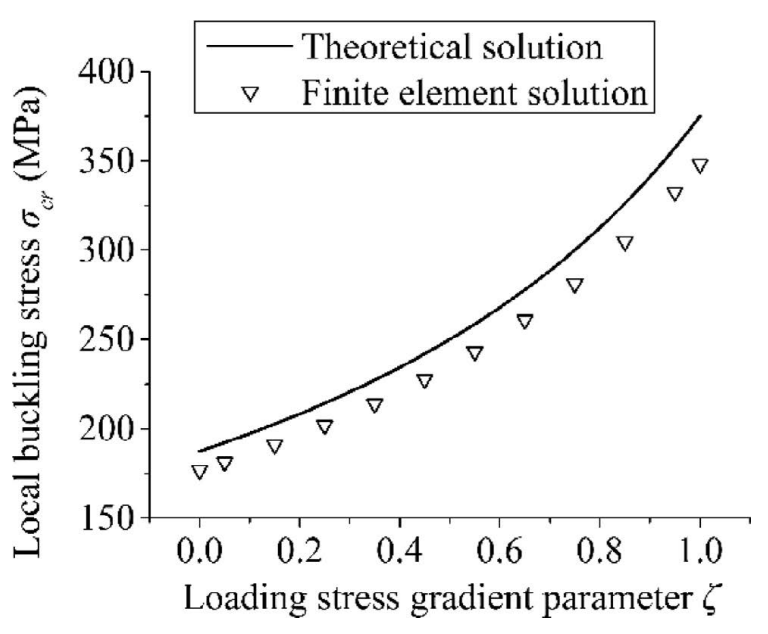

Fig. 6. Comparison of local buckling stress for CC steel plate.

Table 2. Comparison of local buckling stress for CC steel plates.

\begin{tabular}{lccccccc}
\hline$\varsigma$ & $\begin{array}{c}\text { Explicit } \\
N / \mathrm{mm}^{2}\end{array}$ & $\begin{array}{c}\mathrm{FE} \\
N / \mathrm{mm}^{2}\end{array}$ & $\begin{array}{c}\text { Exp/FE } \\
\%\end{array}$ & $\varsigma$ & $\begin{array}{c}\text { Explicit } \\
N / \mathrm{mm}^{2}\end{array}$ & $\begin{array}{c}\mathrm{FE} \\
N / \mathrm{mm}^{2}\end{array}$ & $\begin{array}{c}\text { Exp/FE } \\
\%\end{array}$ \\
\hline 0 & 187.53 & 176.73 & 106.11 & 0.05 & 192.34 & 181.25 & 106.12 \\
0.15 & 202.73 & 191.03 & 106.13 & 0.25 & 214.32 & 201.88 & 106.16 \\
0.35 & 227.31 & 214.03 & 106.20 & 0.45 & 241.97 & 227.69 & 106.27 \\
0.55 & 258.66 & 243.22 & 106.35 & 0.65 & 277.82 & 260.85 & 106.51 \\
0.75 & 300.05 & 281.17 & 106.71 & 0.85 & 326.14 & 304.71 & 107.03 \\
0.95 & 357.20 & 332.46 & 107.44 & 1.00 & 375.06 & 348.11 & 107.74 \\
\hline
\end{tabular}

to $\omega_{1}=\omega_{2}=0$. Substituting these value into Eq. (19) gives the critical aspect ratio $\gamma_{c r}=1.0$. Consequently, the value of critical local buckling coefficient $k_{c r}$ is given as,

$$
k_{c r}=\frac{3.59}{1-0.5 \varsigma}
$$

In the finite element model, one boundary line along the loaded edge of the steel plate $(x=0)$ is completely restrained against all translational degrees of freedom, while the other loaded boundary line $(x=a)$ and the two unloaded boundary lines $(y=0$ and $y=b)$ are restrained against $y$-and $z$-directions translational degrees of freedom. As indicated in Table 3 and Fig. 7, the present solutions of critical stress resultants compare closely with finite element 
Table 3. Comparison of local buckling stress for SS steel plate.

\begin{tabular}{lccccccc}
\hline$\varsigma$ & $\begin{array}{c}\text { Explicit } \\
N / \mathrm{mm}^{2}\end{array}$ & $\begin{array}{c}\mathrm{FE} \\
N / \mathrm{mm}^{2}\end{array}$ & $\begin{array}{c}\text { Exp/FE } \\
\%\end{array}$ & $\varsigma$ & $\begin{array}{c}\text { Explicit } \\
N / \mathrm{mm}^{2}\end{array}$ & $\begin{array}{c}\mathrm{FE} \\
N / \mathrm{mm}^{2}\end{array}$ & $\begin{array}{c}\text { Exp/FE } \\
\%\end{array}$ \\
\hline 0 & 66.53 & 73.90 & 90.03 & 0.05 & 68.24 & 75.80 & 90.03 \\
0.15 & 71.93 & 79.89 & 90.03 & 0.25 & 76.04 & 84.47 & 90.02 \\
0.35 & 80.65 & 89.56 & 90.05 & 0.45 & 85.85 & 95.35 & 90.04 \\
0.55 & 91.77 & 101.94 & 90.02 & 0.65 & 98.57 & 109.42 & 90.08 \\
0.75 & 106.45 & 118.10 & 90.14 & 0.85 & 115.71 & 128.24 & 90.23 \\
0.95 & 126.73 & 140.23 & 90.37 & 1.00 & 133.07 & 147.12 & 90.45 \\
\hline
\end{tabular}

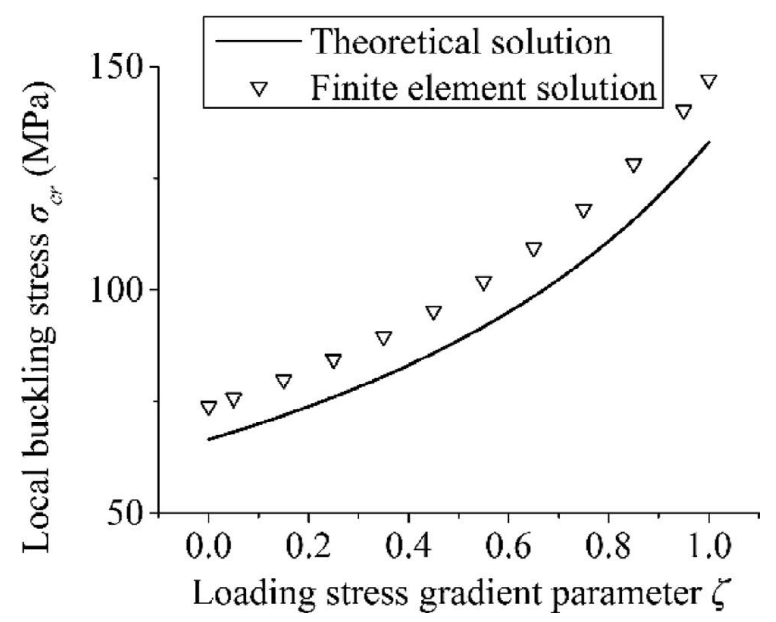

Fig. 7. Comparison of buckling stress for SS steel plate.

eigenvalue analytical results. It can also be concluded that the present explicit solution is highly accurate and can be used with confidence in local buckling analysis. The averaged ratio of explicit solutions to finite element results is $90.12 \%$ and the standard deviation is 0.001 .

\subsection{KS Steel Plate}

The edge restraint depends on the stiffness of adjacent plate fields. Generally, the restraint along the loaded edges is strong while that along the unloaded edges is relative weak. This means the rotational stiffness $k_{x}$ can be assumed as a finite value and the rotational stiffness $k_{y}$ can be set as zero. Therefore, one another special case discussed is the KS steel plate with the loaded edges elastically restrained and the unloaded edges simply-supported.

Finite element simulation was conducted by commercial program ANSYS. The standard springs connecting the boundary nodes to "rigid" support are chosen for the rotational restraining springs. The elastic rotational stiffness of springs along the two loaded edges $(x=0$ and $x=a)$ is set as $10000 \mathrm{~N} \cdot \mathrm{mm} / \mathrm{mm}$. Meanwhile, one boundary line along the loaded edge of the steel plate $(x=0)$ is completely restrained against all translational degrees of freedom, while the other loaded boundary line $(x=a)$ and the two unloaded boundary lines $(y=0$ and $y=b)$ are restrained against $y$ and $z$-directions translational degrees of freedom.

The comparison between the proposed explicit and finite element eigenvalue solutions is given in Table $\mathbf{4}$ and Fig. 8. The averaged ratio of explicit solutions to finite element results is $97.91 \%$ and the standard deviation is 0.007 . It can also be concluded that the present explicit solution is highly
Table 4. Comparison of local buckling stress for KS steel plate.

\begin{tabular}{lccccccc}
\hline \multicolumn{1}{c}{$\varsigma$} & $\begin{array}{c}\text { Explicit } \\
N / \mathrm{mm}^{2}\end{array}$ & $\begin{array}{c}\mathrm{FE} \\
N / \mathrm{mm}^{2}\end{array}$ & $\begin{array}{c}\text { Exp/FE } \\
\%\end{array}$ & $\varsigma$ & $\begin{array}{c}\text { Explicit } \\
N / \mathrm{mm}^{2}\end{array}$ & $\begin{array}{c}\mathrm{FE} \\
N / \mathrm{mm}^{2}\end{array}$ & $\begin{array}{c}\text { Exp/FE } \\
\%\end{array}$ \\
\hline 0 & 108.50 & 111.24 & 97.54 & 0.05 & 111.28 & 114.15 & 97.49 \\
0.15 & 117.30 & 120.31 & 97.49 & 0.25 & 124.00 & 127.13 & 97.54 \\
0.35 & 131.51 & 134.80 & 97.56 & 0.45 & 140.00 & 143.45 & 97.60 \\
0.55 & 149.65 & 153.23 & 97.67 & 0.65 & 160.74 & 164.42 & 97.76 \\
0.75 & 173.60 & 177.35 & 97.89 & 0.85 & 188.69 & 192.34 & 98.10 \\
0.95 & 206.67 & 210.339 & 98.25 & 1.00 & 217.00 & 220.53 & 100.00 \\
\hline
\end{tabular}

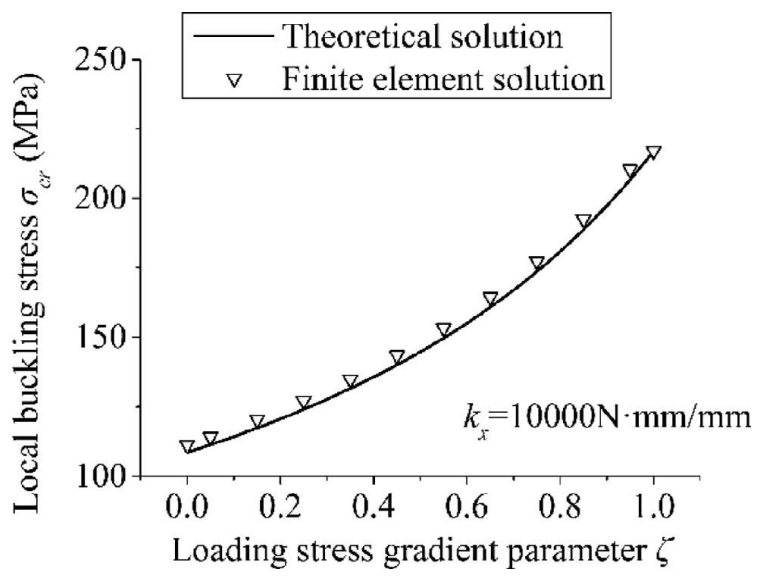

Fig. 8. Comparison of buckling stress for KS steel plate.

accurate and can be used with confidence in local buckling analysis.

\subsection{Discussion}

The proposed analytical model in this research is based, for most part, on sound engineering principles. It is important, though, to stress at this point that the theoretical prediction of the local buckling mechanism of the steel plate in contact with concrete is an extremely complicated matter. In fact, slight differences can be observed between the proposed prediction and the previous research and finite element methods as expressed in Sections 3.1-3.4. As is evident from the derivation of the formulations given in the sections above and the establishment of finite element models, the possible reasons for the differences are listed below:

(1) Simplifications and assumptions were made to the displacement function in this research to reduce the model's complexity to a reasonable level. Different ways of simplifications are also applied by other researchers when aiming for an explicit solution for steel plate under various boundary conditions. The different selections on displacement functions are believed to yield slight difference on the results. This is also the reason for difference between the reduced case for $\mathrm{CC}$ steel plate through the proposed method in this research and the previous findings by analytical approach as described in Section 3.2. As a matter of fact, classical semi-analytical stability analysis of steel plate $^{15)}$ in composite structures states that for many application purposes, the simple sin-functions in both inplane direction as given in Eq. (4) is a more adequate approach for the buckling shape. However, too many unknown coefficients exist in this equation that hinder the derivation of a 
closed-form solution.

(2) The results obtained by finite element method itself may not completely capture the actual local buckling behavior of steel plate. The accuracy of the results in finite element analysis is determined by the choice of an appropriate mesh density. ${ }^{21)}$ While a very fine mesh guarantees the accurate prediction of local buckling, it may as well lead to long computational times. Consequently, both accuracy and efficiency should be taken into account when choosing the mesh size. This can also explain the reason that previous findings by finite element methods from different researchers slightly differ from each other as mentioned in Section 3.2 .

It should be mentioned that, there is no perfect handcalculation design approach available for the local buckling strength of elastically restrained steel plate with combined compression and bending in composite structures yet. However, the results of this study can provide a good understanding of the local buckling behavior of steel plate with elastically restrained edges. Good agreement with a maximum difference of less than $10 \%$ is achieved between the proposed method and the previous research and finite element methods and therefore, should prove useful in design. Although the model presented in this paper may at first appear to be overly complicated, it must be recognized that the studied issue is not simple.

\section{Parametric Study}

As shown in Eq. (26), the explicit local buckling formula is a function of the loading stress gradient parameter $\varsigma$ and the aspect ratio $\gamma$. A parametric study is conducted to illustrate the effects of these factors on the local buckling stress resultants of steel plates.

\subsection{Loading Stress Gradient Parameter $\varsigma$}

The effect of the loading stress gradient parameter on the local buckling stress resultant is plotted in Fig. 9. For a fixed aspect ratio $\gamma=1.0$, as expected, The lower bound and higher bound values in Fig. 9 correspond to the cases of simply-supported and clamped all boundary edges, respectively. Furthermore, the local buckling stress resultants increases with the increase in the loading stress gradient parameter from $\varsigma=0$ to $\varsigma=1$. This means that the steel

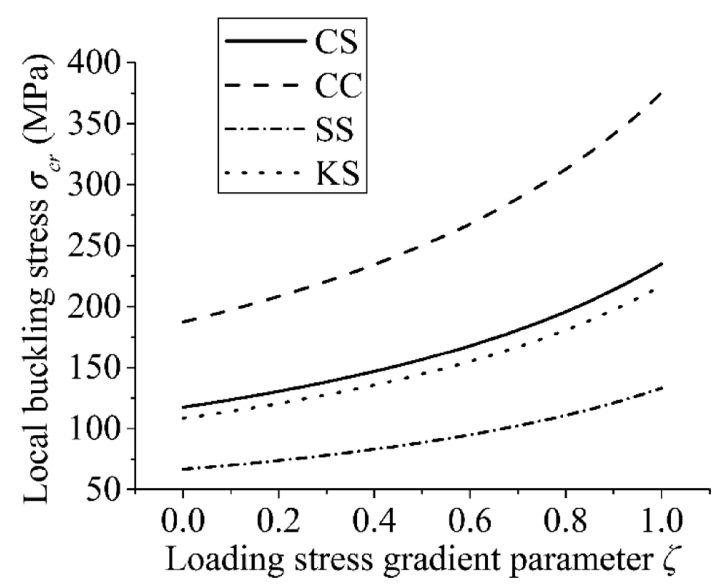

Fig. 9. Effect of loading stress gradient parameter. plate is more vulnerable to local buckling when subjected to uniform compression. Meanwhile, the $\mathrm{CC}$ steel plate is the most sensitive to the change of the loading stress gradient parameter.

\subsection{Aspect Ratio $\gamma$}

The effect of the aspect ratio for local buckling stress resultant of steel plate with different boundary conditions is plotted in Figs. 10-13. The CC and SS steel plates are more sensitive to the change of the aspect ratio. Meanwhile,

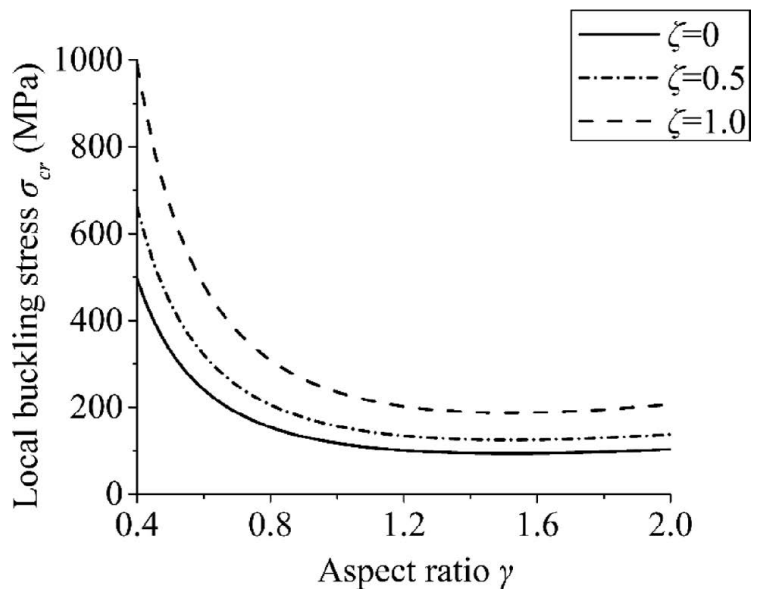

Fig. 10. Effect of aspect ratio for CS steel plate.

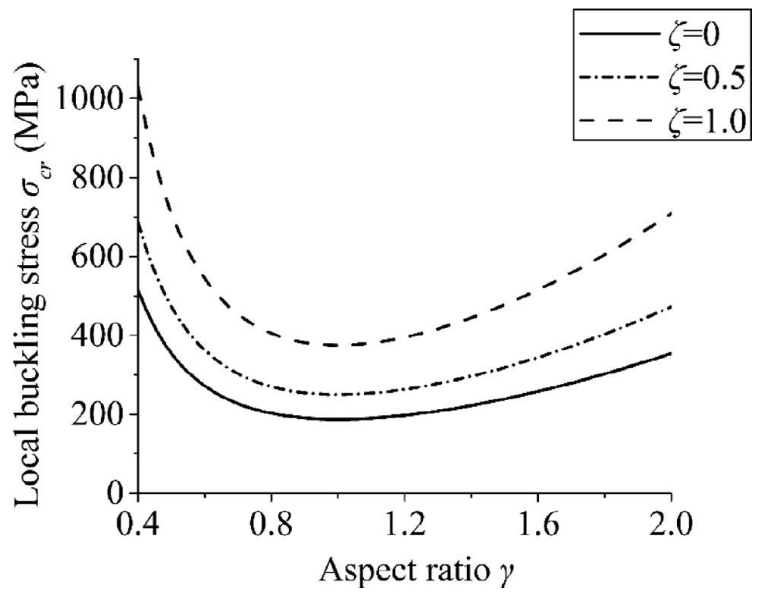

Fig. 11. Effect of aspect ratio for $\mathrm{CC}$ steel plate.

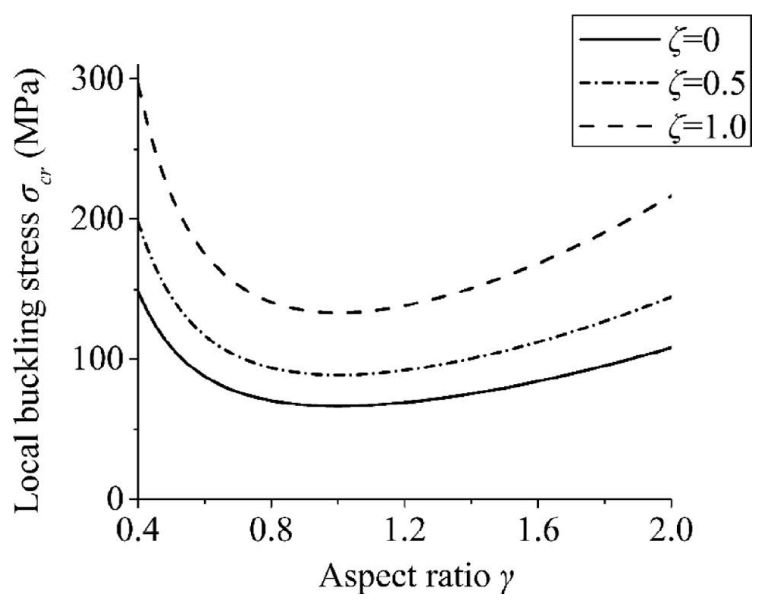

Fig. 12. Effect of aspect ratio for SS steel plate. 


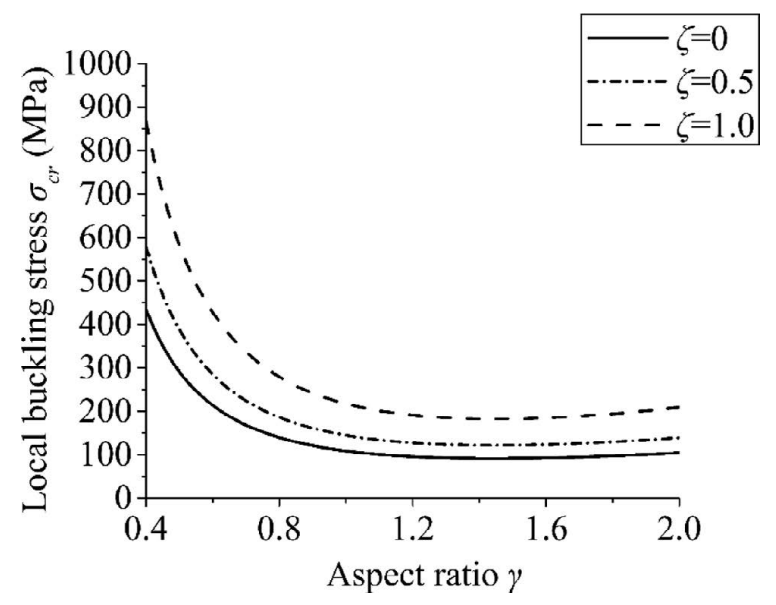

Fig. 13. Effect of aspect ratio for KS steel plate.

the minimum values of buckling stresses of $\mathrm{CS}, \mathrm{CC}, \mathrm{SS}$, and KS steel plates are 93.7 $\mathrm{MPa}, 187.5 \mathrm{MPa}, 66.5 \mathrm{MPa}$, and $91.0 \mathrm{MPa}$, respectively. For CS, CC, SS, and KS steel plates, the aspect ratios of 1.5, 1.0, 1.0, and 1.5, respectively, correspond to the minimum buckling stress resultants.

\section{Summary and Conclusions}

(1) Based on the energy approach and Rayleigh-Rize method, the explicit local buckling eigenvalue solutions for steel plates in composite structures are derived. The unique displacement function which combines the combines sinusoidal and cosine functions is proposed in order to reflect the restraint by concrete.

(2) The derived formulas are reduced to several special cases of steel plates with different boundary restraining conditions. The explicit solutions are compared with both the available solutions in the literature and the finite element results and good agreements are achieved.

(3) Parametric analysis is conducted to evaluate the effects of the loading stress gradient parameter and the aspect ratio on the local buckling stress resultants of steel plates in composite structures with different boundary conditions. It was found that the increase in the loading stress gradient parameter leads to the growth of the local buckling stress, while the value of the aspect ratio greatly affects the local buckling stress resultant.

\section{Acknowledgments}

This work is sponsored by the Natural Science Foundation of Jiangsu Province (Grant No. BK20170685), the National Key Research and Development Program of China (Grant No. 2017YFC0703802), the state Key Laboratory of Subtropical Building Science, South China University of Technology, China (Grant No. 2018ZB26), the Fundamental Research Funds for the Central Universities, and the Priority Academic Program Development of Jiangsu Higher Education Institutions (PAPD).

\section{REFERENCES}

1) S. P. Timoshenko and J. M. Gere: Theory of Elastic Stability, 2nd ed., McGraw-Hill, New York, (1961), 348.

2) P. Qiao, F. Chen, J. Xu and Z. Lu: J. Eng. Mech., 139 (2013), 936.

3) Q. H. Liu, P. Z. Qiao and X. W. Guo: Compos. Struct., 111 (2014), 540.

4) J. H. Dong, X. Ma, Y. Zhuge and J. E. Mills: Steel Compos. Struct., 24 (2017), 697.

5) B. Uy and M. A. Bradford: Eng. Struct., 18 (1996), 193.

6) Q. Q. Liang, B. Uy and M. A. Bradford: J. Struct. Eng., 130 (2004), 443.

7) S. Iwamoto, S. Ochiai and H. Okuda: ISIJ Int., 49 (2009), 139

8) S. Iwamoto, S. Ochiai, H. Okuda and T. Inoue: ISIJ Int., 47 (2007), 930 .

9) H. D. Wright: Struct. Eng., 71 (1993), 209.

10) Y. L. Long, J. Wan and J. Cai: J. Constr. Steel Res., 120 (2016), 70.

11) Y. Qin, J. Y. Lu and S. Cao: ISIJ Int., 57 (2017), 1645.

12) A. Arabzade, H. Moharami and A. Ayazi: Sci. Iran., 18 (2011), 9.

13) A. E. Seif and M. Z. Kabir: Lat. Am. J. Solids Struct., 12 (2015), 2078.

14) E. Jaberzadeh and M. Azhari: Appl. Math. Model., 33 (2009), 1874.

15) C. Mittelstedt: Thin Wall Struct., 45 (2007), 371

16) C. Mittelstedt: Compos. Struct., 91 (2009), 249.

17) J. H. Kang and A. W. Leissa: Int. J. Solids Struct., 42 (2005), 4220.

18) Q. Q. Liang and B. Uy: Compos. Struct., 75 (2000), 479.

19) Q. Q. Liang, B. Uy and J. Y. R. Liew: J. Constr. Steel Res., 63 (2007), 396.

20) R. Q. Bridge and M. D. O’Shea: J. Constr. Steel Res., 47 (1998), 73.

21) P. Kyvelou, L. Gardner and D. A. Nethercot: Eng. Struct., 158 (2018), 28. 